\title{
An investigation into the performance and diagnostics from different chordal integration schemes in asymmetric flow.
}

ARNOULD, P.

2020 


\section{Journal Pre-proof}

An Investigation into the Performance and Diagnostics from Different Chordal Integration Schemes in Asymmetric Flow

Patrick Arnould

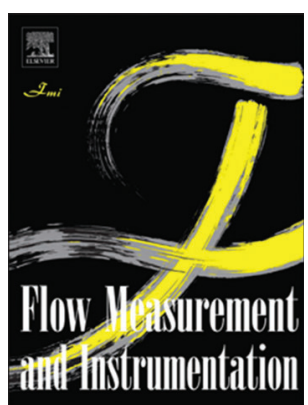

PII: S0955-5986(19)30183-9

DOI: https://doi.org/10.1016/j.flowmeasinst.2019.101644

Reference: JFMI 101644

To appear in: Flow Measurement and Instrumentation

Received Date: 9 May 2019

Revised Date: 3 September 2019

Accepted Date: 30 September 2019

Please cite this article as: P. Arnould, An Investigation into the Performance and Diagnostics from Different Chordal Integration Schemes in Asymmetric Flow, Flow Measurement and Instrumentation, https://doi.org/10.1016/j.flowmeasinst.2019.101644.

This is a PDF file of an article that has undergone enhancements after acceptance, such as the addition of a cover page and metadata, and formatting for readability, but it is not yet the definitive version of record. This version will undergo additional copyediting, typesetting and review before it is published in its final form, but we are providing this version to give early visibility of the article. Please note that, during the production process, errors may be discovered which could affect the content, and all legal disclaimers that apply to the journal pertain.

(C) 2019 Elsevier Ltd. All rights reserved. 


\title{
An Investigation into the Performance and Diagnostics from Different Chordal Integration Schemes in Asymmetric Flow
}

\author{
Patrick Arnould, Oil \& Gas Authority, Robert Gordon University
}

\begin{abstract}
The purpose of the work is firstly to explore the proposal from a previous North Sea Flow Measurement Workshop paper that increasing the number of chordal locations above 4 yields little improvement in the performance of ultrasonic flow meters. Secondly the work examines the relationship between profile factor and symmetry on theoretical velocity profiles and how that changes with an increased number of chordal locations. Finally, the benefits of more paths for the purposes of swirl cancellation are reviewed, particularly when axial asymmetry and swirl are present concurrently.

It is found that in the presence of axial asymmetry there is a significant performance improvement to be had with an increased number of chordal locations.

A relationship between profile factor and error is also apparent. The interpolation polynomial and weight function are used to visualize the quadrature and explain the correlation. The theory that more chordal locations will improve the correlation between error and profile factor is investigated, however initial results suggest this is not true.

The theory behind swirl cancellation is explained and the velocity profile diagnostics of common designs in the presence of swirl and axial asymmetry are shown. Examples of how the user can be fooled into thinking all is well in the presence of both axial asymmetry and swirl are also shown. The sensitivity of some designs to swirl is also examined.

The modelling suggests that in cases where swirl and axial asymmetry are present together and non-axial velocity components are not removed by swirl cancellation or flow conditioners, a change in traditional velocity profile diagnostics cannot be used to indicate the magnitude of the error.
\end{abstract}

\section{INTRODUCTION}

One of the findings from the 2012 North Sea Flow Measurement Workshop paper 'Limits of Achieving Improved Performance from Gas Ultrasonic Meters and Possible Solutions' [1] was that increasing the number of paths beyond 4 has little effect on the integration.

In the paper, the power law profile was used to make this assertion and a specific type of Gaussian quadrature. It appears that the original intent may have been to illustrate whether more chords offer much improvement in the integration. It was therefore decided to model the effect of more chords on the integration in the presence of axial asymmetry and no swirl for two different methods of Gaussian quadrature and see if this conclusion still holds true.

The correlation of profile factor with meter error for these asymmetric profiles will also be reviewed including why the correlation appears to work and the effect of more chords will also be shown.
The performance improvements possible with more paths are also considered. The word 'path' should not be considered synonymous with 'chord' because this can lead to confusion. For example, there exist 8 path 4 chord meters with 2 paths per chord with the paths crossed for the purposes of swirl cancellation. These meters offer significant performance benefits over their 4 path 4 chord siblings and this should not be overlooked [2]. This design of meter and the issues that arise with an unknown combination of swirl and axial asymmetry will be discussed.

\section{THE EFFECTS OF AXIAL ASYMMETRY}

\subsection{Background}

Axial velocity profiles describe the distribution of velocity in the axial direction (i.e. straight down the length of the pipe). There are assumed to be no components in any other direction, or in other words no swirl. Similarly, axial asymmetry means an axial velocity profile which is not symmetrically distributed around the pipe centre. In real velocity profiles asymmetry and swirl can exist together in various proportions, although in theory it is possible to have a perfectly symmetrical profile with swirl or an asymmetric axial profile with no swirl. The modelling in this section is concerned with the distribution of axial velocity, how it affects the performance and diagnostics of chordal meters and how this changes with more chordal locations.

This scenario does exist in real life; for example, a flow conditioner can remove swirl components but leave persistent asymmetry in the profile which slowly decays.

It is necessary to define what is meant by the terms chord and path to lessen confusion because the terminology is not necessarily consistent between manufacturers. A detailed explanation of this issue can be found in [10]. In this paper, a 'path' is the straight line through the fluid connecting transducers. A 'chord' is the straight line connecting two points on the circumference of a circle. A 'chordal path' is the path (or paths) that lines up with a chord in a cross-sectional view of the meter. The term 'chordal location' describes the height of the chord from the pipe centre. The chords are illustrated below in Fig. 1.1 as A - D. The chordal paths can be visualised by imagining a view from the top of the meter looking down as in Fig. 1.2 or 1.3. One would then see the chordal paths for A (and beneath them B, C and D). At each chord, there could be only a single path with a positive or negative transducer inclination angle $\theta$, or there could be two paths on the same chord or chordal location. 


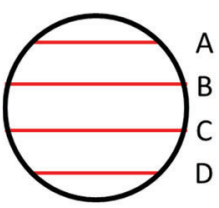

Fig. 1.1-4 chords

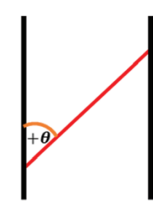

Fig. $1.2-$

Single path per chord

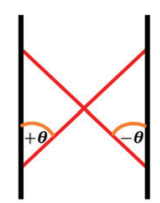
on the same chord
Fig. 1.3 - Two paths

Chordal ultrasonic flow meters make use of Gaussian quadrature to integrate the velocity profile. Quadratures are designed to calculate the definite integral of a function with only a select few samples of the function on which to base its calculation. The weights which many are familiar with are derived by integrating interpolation polynomials. We can construct the interpolation polynomial and use it to illustrate why the meter is affected in a certain way when errors begin to develop. It is the interpolation polynomial which gives the meter an inherent ability to cope with some asymmetry in the axial profile.

The following equations illustrate the basics of Gaussian quadrature adapted for use in ultrasonic meters (USMs). Note that the equations are written for illustrative purposes assuming a meter with a radius of unity. Be aware there is a further modification required to adapt the weights to operate over a different interval. Imagine an infinite number of chords one ever so slightly below the previous, each determining the average velocity along the chord multiplied by chord length, or the line integral. If those values were plotted you could visualise the function of chordal line integrals. When this function is integrated w.r.t. height and the result divided by cross-sectional area this gives us the mean velocity $\bar{V} \cdot f(x)$ in Eq. (1) is the true function of chordal line integrals and this function defines the mean chordal velocity multiplied by chord length or $\bar{v}(x) L(x)$ at each height ??. However, we are only sampling at a few chords so we can only approximate this function, the interpolation polynomial fills in the gaps for us. To build the interpolation polynomial the Lagrange polynomial is used along with samples of the true function at a number of discrete points. The integration of the Lagrange polynomials provides us with the weighting factors $A_{k}$ and they include the modification to operate in a circular domain and return mean velocity.

$$
\begin{aligned}
& \bar{V}=\frac{\int_{-1}^{1} f(x) d x}{\pi} \approx \frac{\int_{-1}^{1} P(x) d x}{\pi} \\
& =\frac{\sum_{k=1}^{n} \bar{v}\left(x_{k}\right) L\left(x_{k}\right) \int_{-1}^{1} l_{k}(x) d x}{\pi}=\sum_{k=1}^{n} \bar{v}\left(x_{k}\right) A_{k}
\end{aligned}
$$

The Lagrange polynomials are formulated as per Eq. (2).

$$
l_{k}(x)=\prod_{\substack{r=1 \\ r \neq k}}^{n} \frac{x-x_{r}}{x_{k}-x_{r}}
$$

As shown above, calculating the weighting factors and applying them in this way integrates the interpolation polynomial exactly, not necessarily the true function. We can get closer to the true function with more chordal locations because there are more points for the interpolation polynomial to base its prediction on as is shown later.

The above quadrature rule would integrate an order $\mathrm{n}-1$ polynomial with $\mathrm{n}$ samples of the true function. Gaussian quadrature is a specific type of quadrature that is more powerful than the conventional quadrature rule shown above. In fact, with $\mathrm{n}$ samples this method can integrate a polynomial of the order $2 n-1$. The chordal locations are chosen as the zeroes of a specific polynomial depending on the quadrature type, e.g. Jacobi, Legendre, Chebyshev. Each polynomial has a corresponding weight function which, through the principle of orthogonal polynomials, permits integration of a higher order function with a given number of points. It also expands the capability of the quadrature to integrate functions which are not polynomials. Eq. (3) shows the addition of the weight function.

$$
\begin{aligned}
& \bar{V}=\frac{\int_{-1}^{1} f(x) d x}{\pi} \\
& =\sum_{k=1}^{n} \bar{v}\left(x_{k}\right) L\left(x_{k}\right) \frac{\int_{-1}^{1} l_{k}(x) w(x) d x}{\pi w\left(x_{k}\right)} \\
& =\sum_{k=1}^{n} \bar{v}\left(x_{k}\right) A_{k}
\end{aligned}
$$

The weights are divided by the weight function evaluated at the associated chordal location when used in this way which results in $\bar{v}\left(x_{k}\right) L\left(x_{k}\right) /\left(\pi w\left(x_{k}\right)\right)$ being used as the basis for the interpolation polynomial. Note also that $L(x)=2\left(1-x^{2}\right)^{0.5}$ and is not a polynomial. The Lagrange interpolation polynomial cannot interpolate functions which are not polynomials, but this problem can be solved through clever selection of $w(x)$, e.g. if $w(x)=\left(1-x^{2}\right)^{0.5}$.

This example must be extended further to fully appreciate how Gaussian quadrature reduces the problem. Assuming our above definition of $L(x)$ and also our selection of $w(x)$ if we further assume that $\bar{v}(x)=$ $a(x) b(x)+r(x)$ and $a(x)$ is the polynomial upon which the quadrature is based, because $a(x)$ is orthogonal w.r.t. $w(x)$ then $\int_{-1}^{1} a(x) b(x) 2 w(x) / \pi d x=0$ and we are only integrating $r(x) 2 w(x) / \pi$. The interpolation polynomial also needs to account for the lack of the $a(x)$ and $b(x)$ functions which is captured in the design of the quadrature. The locations of the chords are the zeroes of the polynomial upon which the quadrature is based, therefore each sample which is fed into the interpolation polynomial is based on $2 r(x) / \pi$ hence it can replicate the reduced function. This function will be of a lesser order than $\bar{v}(x)$ therefore using this method we can integrate a higher order function with a given number of points.

We can also compare the interpolation polynomial with the function of chordal line integrals as per Eq. (4).

$$
f(x) \approx w(x) \sum_{k=1}^{n} \frac{\bar{v}\left(x_{k}\right) L\left(x_{k}\right)}{w\left(x_{k}\right)} l_{k}(x)
$$


2.2 Modelling the Effects of Axial Asymmetry on Transit Time USMs

For the purposes of modelling the effects of axial asymmetry the measurement principle of the transit time ultrasonic flow meter can be modelled using existing mathematical 2D velocity profile functions as explained in [3], [5] and [6]. The line integral is approximated numerically across these functions and used to represent the path measurements of velocity. The line integral allows computation of the mean velocity across a chord of the profile function, which is a simplified representation of the velocity being inferred from a measurement of upstream and downstream transit times. The mean velocity for each of the chords at defined locations are used according to the principles of Gaussian quadrature which models the meter output.

Since the profile functions are defined they can be integrated numerically or analytically to allow calculation of the true mean velocity for the velocity profile function. The mean velocity output from the quadrature can then be compared with the true mean and the quadrature error, or percentage difference between these two values, determined.

The model gives us the path velocities from which traditional profile factor and symmetry ratio can be calculated. The asymmetric profiles are specified in polar coordinates and to perform the line integral one must calculate the radius and angle from the horizontal for each point in the path. By including an angular offset, the profile can be rotated which yields a great deal more velocity profile diagnostic information.

An illustration of the rotation of the profile is given in Table 1. This is an example velocity profile contour plot (high velocity is blue, low is brown), and it can be seen this allows us to model rotation of the meter. One can expect different integration errors at different angles as reported in [6].
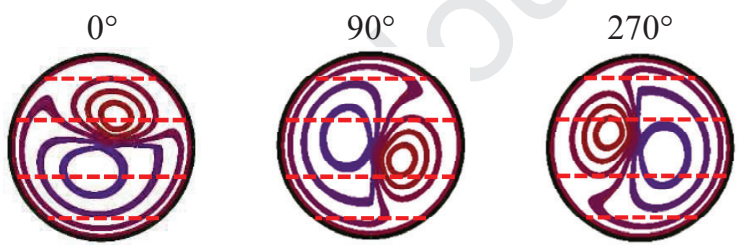

Table 1 -Rotation of the velocity profile (dashed lines indicate the chordal paths)

To model the performance and diagnostic changes more chordal locations can yield $4,5,6,7$ \& 8 chordal locations were used for both quadrature types.

The model includes the 10 profile functions mentioned in paper [4] which are based on the Salami profiles but the asymmetry term has been reduced as the author understands to make the errors match closer with those seen in laboratory tests. The power law profile has also been implemented. There is also the facility to perform line integrals very closely spaced from top to bottom of the profile function and output the data which allows visualisation of the profile and comparison with the interpolation polynomial on which the quadrature is based.

To determine the weights and nodes (or locations) of the quadratures the polynomial definition and the associated weight function are required. This work focusses on Gauss-Jacobi (also known as GaussChebyshev for our configuration of the Jacobi polynomial) and Gauss-Legendre since it is understood these represent integration schemes used by chordal meters seen during inspections performed by the author.

Determining the nodes and weights can be a lengthy process. However pre-calculated nodes and weights are available online [7] or in papers [3].

\subsection{Results - Power Law Profile}

We first show the performance of the $4-8$ chordal location Gauss-Jacobi and Legendre quadratures on the power law profile. The range of $n$ values was $6 \leq n \leq$ 11 , representing a Reynolds number range of $15000 \leq$ $R e \leq 15 \times 10^{6}$. This covers a range of profile factors and for 4 chord Gauss-Jacobi the range was 1.21 - 1.11.

Note that the Legendre implementations in this section and section 2.4 use correction factors to account for an oddity when this quadrature is used in a circular domain. Use of the correction factor results in weights which sum to 1 and merely shifts all errors in the negative direction. One manufacturer has explained this adjustment is performed in their meters [4] and hence this make the comparison with Gauss-Jacobi fairer.

Errors seen at the first calibration of a new meter will be due to many factors including chordal locations not being exactly where required for the quadrature, slight geometry measurement errors in the meter internal diameter, path lengths and path angles and the inherent error due to the quadrature's ability to accurately integrate the velocity profile. The below results can be thought of as the change in this inherent error in the quadrature which would only be visible at the first calibration of a new meter. Thereafter any error from this component is effectively calibrated out.

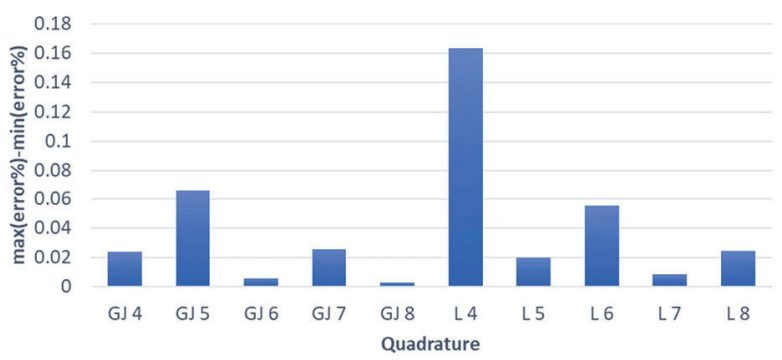

Fig. 2 - Error span with increasing chordal locations (GaussJacobi \& Gauss-Legendre)

Fig. 2 shows the span of errors across the range of $n$ on the power law profile. We note that in general the span of error is decreasing as the number of chords increases, although for the Gauss-Jacobi the span is reduced with an even number of chords (i.e. 4 chords are better than 5 and 6 are better than 7) and the reverse appears evident for Legendre. 


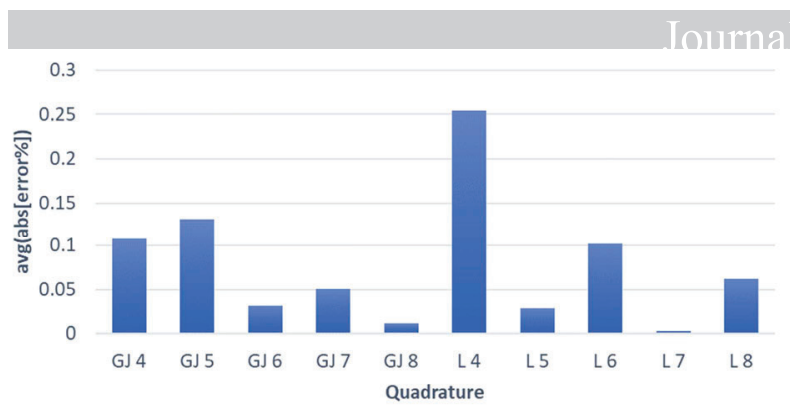

Fig. 3 - Average absolute error with increasing chordal locations (Gauss-Jacobi \& Gauss-Legendre)

Fig. 3 shows the average absolute error across the range of $\mathrm{n}$ on the power law profile. The Legendre quadrature shows a significant improvement in performance as the number of chordal locations increases whereas Gauss-Jacobi shows less of an improvement. The results reported by $\mathrm{K}$. Zanker [1] are similar to those above in that we have a small increase in performance, $\sim 0.1 \%$ to $\sim 0.01 \%$ as the number of chordal locations increase for Gauss-Jacobi. However, those results do not consider the performance of the quadrature with asymmetric profiles which now follows.

\subsection{Results - Asymmetric Profile Functions}

Note that a variation of the metric 'orientation sensitivity factor' defined by P. Moore et al. [3] has been used which in this case is the difference between the maximum and minimum $\%$ errors at different orientations. Note 37 different orientations were used for each profile in $5^{\circ}$ increments from $0^{\circ}$ to $180^{\circ}$.

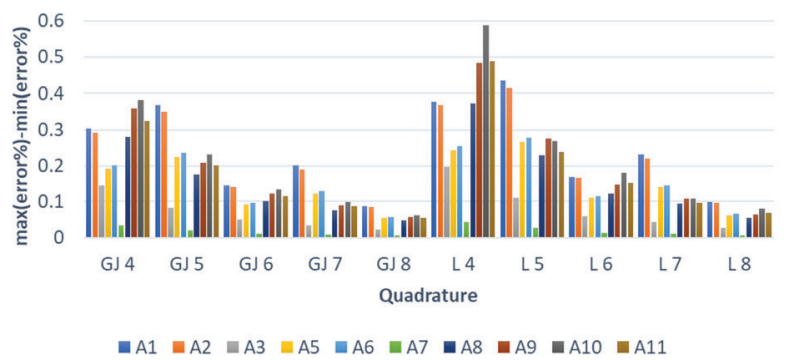

Fig. 4 - Error span with increasing chordal locations

Fig. 4 shows that there is a significant improvement across the different profiles with a reduction in the error span of $0.2 \%-0.3 \%$ being achieved on many of the profiles when comparing 4 to 8 chordal locations.

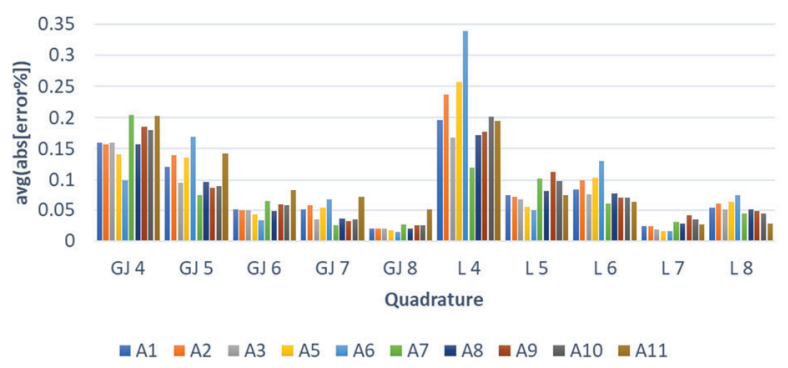

Fig. 5 - Average absolute error with increasing chordal locations

Fig. 5 shows that the average absolute error is reducing as the number of chordal locations increases by $\sim 0.15 \%$ for Gauss-Jacobi and $\sim 0.2 \%$ for Gauss-Legendre.
There are some practical problems with introducing more chords which have been described in [1]. For example, with more chords the velocity profile disturbance created by the transducer cavity increases which itself causes problems with the integration. There may also be problems with interference between paths or unintended reflections. It is understood that these problems are more evident in smaller meters than larger meters and that larger USM's in water applications do make use of a greater number of chordal locations. The size of meter for which these problems become unsurmountable with different numbers of chordal locations is not presently known, but presumably as the technology develops further these challenges will become less of an issue. It is also worth considering the potential for offering increased chordal locations in larger sizes only as these meters will likely be measuring a greater value of product and therefore reductions in sensitivity to axial asymmetry become more fiscally important.

Note that manufacturers in some cases also include diagnostic corrections in the meter software which may reduce the effect of the above. These corrections when employed for axial asymmetry correction can be thought of as an alternative to more chordal locations. These corrections are commercially sensitive and are not divulged. When we have differences between profiles at calibration and in service these corrections may be in use to a lesser or greater degree depending on the difference. However, there is a disadvantage to correcting for axial asymmetry this way which is discussed in part 4 .

We shall now explore correlations between diagnostics and integration errors caused by axial asymmetry only (no swirl present).

\subsection{Diagnostic Correlations - Axial Asymmetry}

The relationship between profile factor and integration error was reviewed using the data from the asymmetric profile functions at different orientations. Note that profile factor is calculated as the sum of the inner path velocities divided by the sum of the outer path velocities.

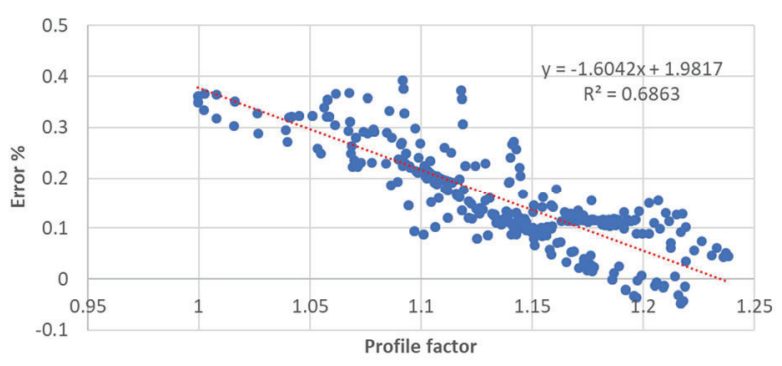

Fig. 6 - Correlation between integration error and profile factor on the asymmetric profiles (4 chord Gauss-Jacobi)

Fig. 6 shows a relationship between error and profile factor as reported in [4]. The \% change in error per $\%$ change in profile factor (sensitivity) from the above is $0.016 \%$ per $1 \%$ increase in profile factor. Note this represents a theoretical correction for asymmetry only, no swirl. Figs. 7A and 7B show the same data split into two plots, the profile function each data point belongs to is indicated. 


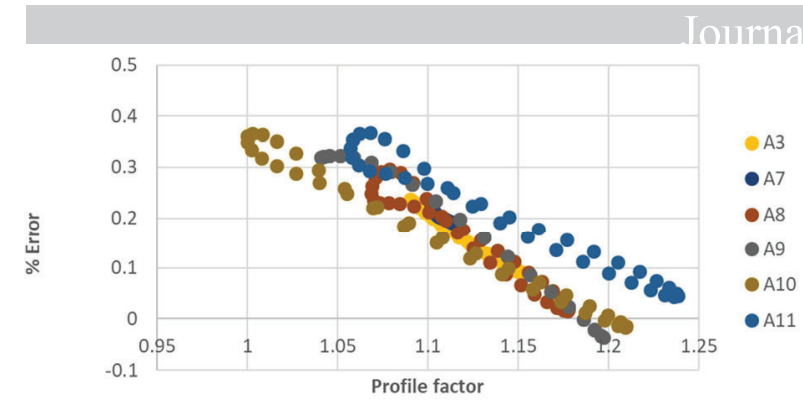

Fig. 7A-Correlation between integration error and profile factor for some asymmetric profiles (4 chord Gauss-Jacobi)

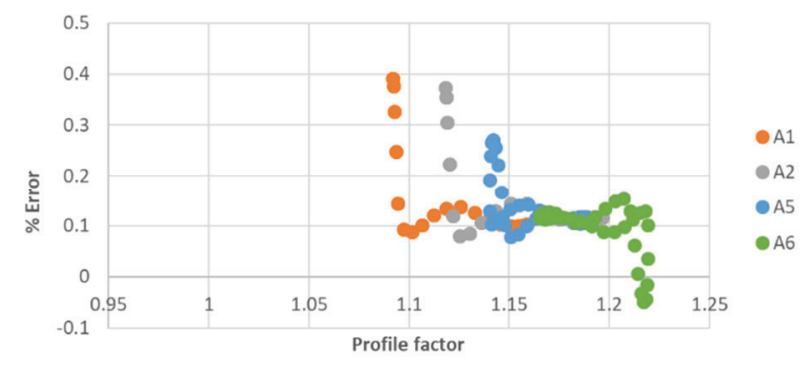

Fig. 7B-Lack of correlation between integration error and profile factor for some asymmetric profiles (4 chord GaussJacobi)

Fig. 7A shows that there are some profile functions for which the correlation works well, e.g. A3, A7, A8, A9, A10 and A11. Fig. 7B shows that there are some profiles that it does not work for e.g. A1, A2, A5 and A6. Using the model, the function of chordal line integrals was output and compared with the interpolation polynomial to understand why the correlation works for some of the profiles and not others.

Figs. $8 \mathrm{~A}$ and $8 \mathrm{~B}$ show profile $\mathrm{A} 8$ at angles of $95^{\circ}$ and $0^{\circ}$ respectively compared with the Gauss-Jacobi 4 chord interpolation polynomial. For each chart the interpolation polynomial is calculated as per equation (4) with samples from the velocity profile function with the associated angular offset applied. The upper chart corresponds to an error of $+0.02 \%$ and it is clear the interpolation polynomial is a fair fit. The lower chart shows the interpolation polynomial overestimating the mean chordal velocity and the error has increased to $+0.28 \%$.

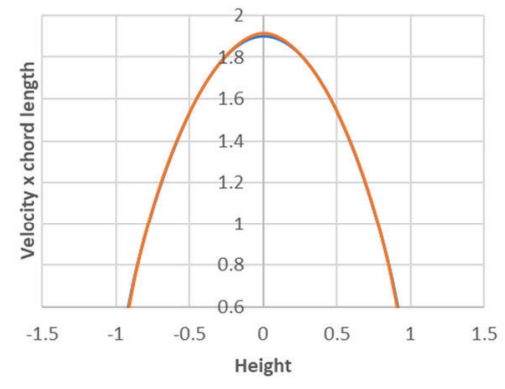

—interpolation polynomial_—A8@ $95 \mathrm{deg}$

Fig. 8A-Comparison of mean chordal velocity and interpolation polynomial for profile $A 8$ at $95^{\circ}$.

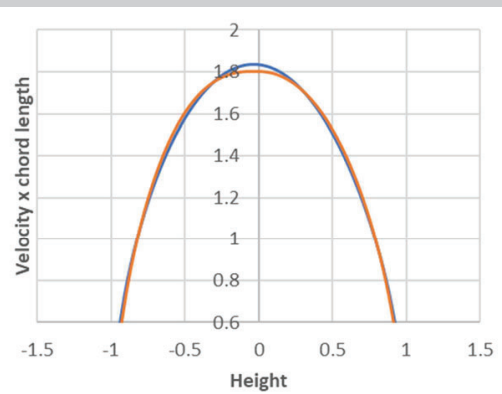

—interpolation polynomial —A8@0 deg

Fig. 8B-Comparison of mean chordal velocity and interpolation polynomial for profile $A 8$ at $0^{\circ}$.

It appears that the reason for the correlation is that as the profile gets flatter (and hence profile factor decreases) there appear to be changing gradients between the inner chords which the interpolation polynomial is unable to predict and causes an overestimate around the pipe centre. There is also a lesser degree underestimate between the inner and outer chords but this does not counter the overestimate around the centre and results in a positive error.

A comparison of the true mean chordal velocity with the interpolation polynomial was also performed for profile A1, shown in Fig. 9. It was found there is a discontinuity present which is causing a larger than expected error across the range and should not appear in real life. This has been investigated in previous work [3]. The chordal velocity function was plotted for all profile functions at $0^{\circ}$ which demonstrated that $\mathrm{A} 2$ and $\mathrm{A} 5$ contain a similar discontinuity to that shown below. A6 does not appear to have the same discontinuity but it is detailed in [3] that the angular function which forms part of the profile function does not have a continuous derivative w.r.t. $\theta$, so it could be argued it may not be realistic.

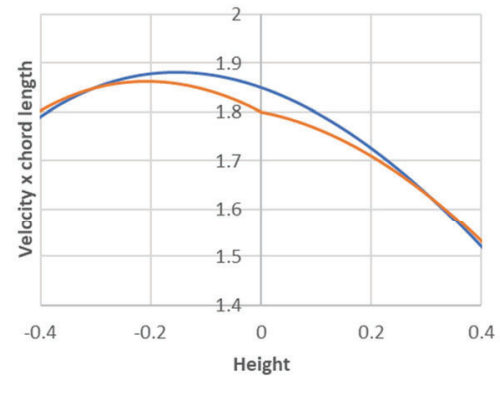

—interpolation polynomial_—A1@0 deg

Fig. 9 - Comparison of mean chordal velocity and interpolation polynomial - A1

The correlation was rechecked with the profiles of questionable validity removed. It was found that the sensitivity was $\sim-0.018 \%$ with an $r^{2}$ of 0.84 . The GaussLegendre profile factor correlation was generally better in terms of fit than Gauss-Jacobi for the same number of chords, for example at 4 chords $r^{2}$ was 0.93 . The only exception to this rule was for 5 chords where GaussJacobi achieved an $r^{2}$ of 0.92 and Legendre achieved 0.84 . Gauss-Legendre appeared slightly more sensitive to changes in profile factor for a given number of chords, for example at 4 chords sensitivity was $-0.025 \%$. 
The integration error was plotted as a function of symmetry ratio to see if a correlation was evident, see Fig. 10. Note that the symmetry ratio is calculated as the sum of the upper path velocities divided by the sum of the lower path velocities.

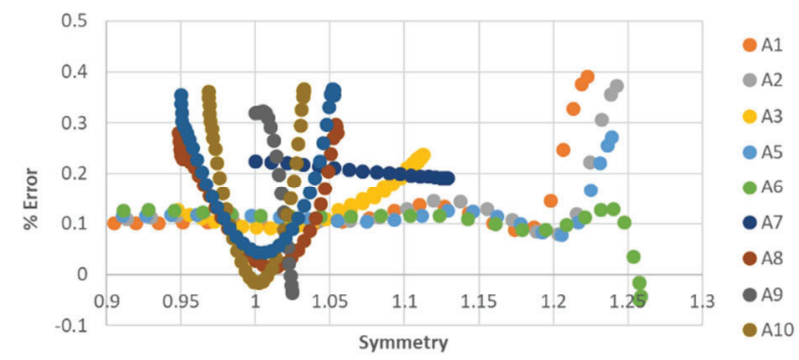

Fig. 10 - Correlation between integration error and symmetry ratio on the asymmetric profiles (4 chord Gauss-Jacobi)

From Fig. 10 it appears that there is not a useful correlation relating errors due to axial asymmetry with the symmetry ratio.

Fig. 11 shows the relationship between error and profile factor for the 7 and 8 chord Gauss-Jacobi quadrature and test if the relationship between profile factor and error becomes stronger with more chordal locations.
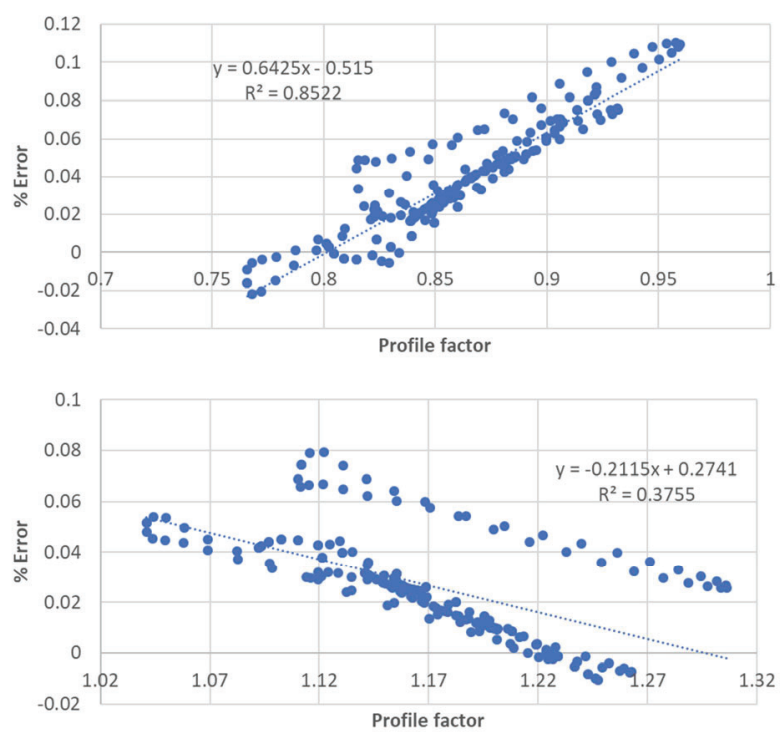

Fig. 11 - Correlation between integration error and profile factor on the asymmetric profiles for 7 chord (top) and 8 chord (bottom) Gauss-Jacobi

Fig. 11 shows that the correlation is only marginally improved with 7 chords and is not improved with 8 chordal locations. From the trends $r^{2}$ increased from 0.84 with 4 chordal locations to 0.85 with 7 and dropped to 0.38 with 8 . For Gauss-Legendre generally $r^{2}$ decreased with the number of chords from 0.93 to 0.57 with 8 chords, the exception being at 7 chords in which $r^{2}$ was 0.87

The total spread of error across the range of profile factors is vastly reduced with 8 chords and sensitivity is $-0.002 \%$ for Gauss-Jacobi and $\sim-0.003 \%$ for GaussLegendre, i.e. the integration scheme is less sensitive to changes in profile factor caused by axial asymmetry.

\subsection{Background}

Swirl can be thought of as any non-axial component of velocity and has a separate effect to axial asymmetry on the measurement. The issue with swirl arises from an assumption in Eq. (5):

$$
t_{A B}=\frac{L}{c+v \cos \sigma} \quad t_{B A}=\frac{L}{c-v \cos \sigma}
$$

The measurement principle is simply time equals distance divided by speed. Remember that the meter does not measure velocity, it measures transit time and it infers velocity. To calculate the expected speed of the pulse used in the formulation of the equation for velocity we take the speed of sound in the fluid and add/subtract a correction for the component of velocity in the direction of the path. A vector diagram illustrating this is shown in Fig. 12:

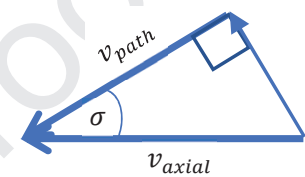

Fig. 12 - Path component and axial velocity vectors

$$
\cos \sigma=\frac{v_{\text {path }}}{v_{\text {axial }}}
$$

To be able to infer axial velocity from the difference in transit times the ratio of the path to the axial velocity must be equal to the cosine of the angle between them. If there is swirl in the profile there will be another velocity helping or retarding the pulse. Consequently $v_{\text {path }}$ will be higher or lower than expected and these equations are no longer true leading to an error in the velocity measurement.

Three different chordal meter designs are referenced within this section. According to BS-7965:2013 [12] these three types are as follows, multi-path parallel chord, multi-path parallel crossed chord and multi-path in-plane crossed chord. These definitions will be used minus the multi-path description. The parallel crossed chord configuration which has been used in this paper has $\mathrm{A}$ and $\mathrm{C}$ chords with a transducer inclination angle of $-60^{\circ}$ and $\mathrm{B}$ and $\mathrm{D}$ chords at angle of $+60^{\circ}$. The parallel chord configuration has an inclination angle of $+60^{\circ}$ for all chords.

\subsection{Swirl Cancellation}

For some time, there has been a meter design in production which can combat the issue of swirl from first principles using swirl cancellation, although it appears uptake in the fiscal measurement side of the oil and gas industry has been slow and the author has only seen one so far during inspections. Note that true cancellation of asymmetric swirl will only occur if the crossed paths are at the same elevation.

The mechanics of the swirl cancellation employed by the in-plane crossed chord design are illustrated below in a worked example using only basic trigonometry and vectors. If we assume we have a given axial velocity, say $15 \mathrm{~m} / \mathrm{s}$ and say we have a swirl velocity of $2 \mathrm{~m} / \mathrm{s}$. We 
Joumal Pre-proof

construct a new combined velocity vector as shown in Fig. 13.

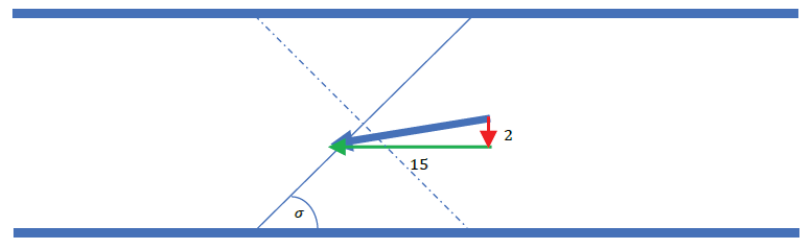

Fig. 13 - Formulation of the combined velocity vector (positive path angle)

The swirl angle $\emptyset_{s w}=\tan ^{-1}\left(v_{\text {swirl }} / v_{\text {axial }}\right)=7.6^{\circ}$ and our new combined velocity magnitude

$v_{\text {comb }}=\left(v_{\text {axial }}{ }^{2}+v_{\text {swirl }}{ }^{2}\right)^{0.5}=15.13 \mathrm{~m} / \mathrm{s} . \quad \mathrm{We}$ can show the effect this combined velocity would have on the path velocity. To do this we calculate the component of the combined velocity vector in the direction of the path as shown in Fig. 14

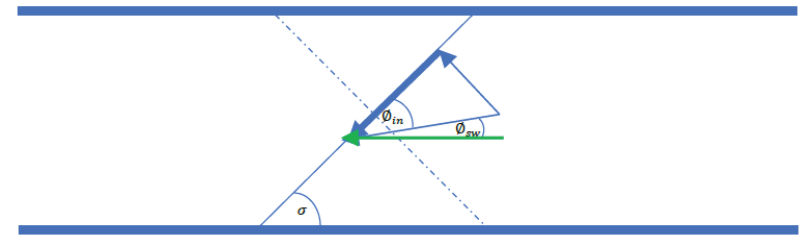

Fig. 14 - Calculation of the path velocity vector (positive path angle)

The angle between the combined velocity vector and the path itself, termed here the interception angle $\emptyset_{\text {in }}$ is calculated by subtracting the swirl angle $\emptyset_{s w}$ from the path angle $\sigma$ and we find it is $52.4^{\circ}$. The component of velocity in the direction of the path $v_{\text {comb path }}=$ $v_{\text {comb }} \cos \emptyset_{\text {in }}=9.23 \mathrm{~m} / \mathrm{s}$. The USM measures the time of flight along the path which will obviously be heavily dependent on this velocity and the conversion back to an axial velocity is handled in the formation of the velocity equation. We must now convert our answer back to an axial velocity to mimic the USM and we can only use the path angle to do this, hence the velocity error creeps in. In our simplified example, axial velocity for this path is as follows:

$$
v_{\text {axial_path }}=v_{\text {comb_path }} / \cos \sigma=18.46 \mathrm{~m} / \mathrm{s} \text {. }
$$

We now repeat this for a second path which crosses the first and is on the same chord as shown in Fig. 15. This should experience the same swirl velocity in our simplified example.

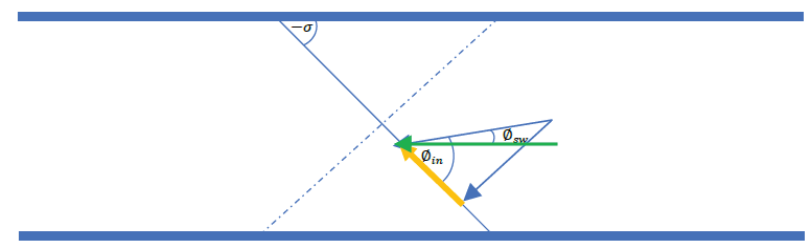

Fig. 15 - Calculation of the path velocity vector (negative path angle)

For this path, the path angle is $-\sigma$ otherwise the calculations are performed in the same way as before. We have a reduced path component of $5.77 \mathrm{~m} / \mathrm{s}$ because of the larger interception angle $\left(-67.6^{\circ}\right)$. Finally, the component of velocity in the direction of the path is converted to give an inferred axial velocity for this path as before giving $11.54 \mathrm{~m} / \mathrm{s}$. The effect on each of these paths is equal in magnitude but opposite in sign, so if we now average the two axial velocities we arrive at the true axial velocity of $15 \mathrm{~m} / \mathrm{s}$.

Another feature of the design is the ability to infer the swirl velocity from the difference in path velocities. Geometrically this can be visualised as per Fig. 16. This has been constructed from the above example, the two bold arrows on the left are the path components of velocity from Fig. 14 and Fig. 15.

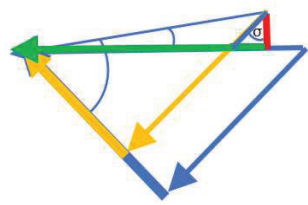

Fig. 16-Calculation of the swirl velocity from half the difference in path velocities

We can therefore take our difference in path velocities which is $18.46-11.54=6.92$. Referring to Fig. 16 the swirl component (at a right angle to the axial velocity vector) is part of a small right-angled triangle and is adjacent to the known angle, the side opposite the angle is the half difference in path velocities so the swirl is simply calculated from $v_{\text {swirl }}=6.92 /(2 \tan \sigma)=2$ $\mathrm{m} / \mathrm{s}$.

\subsection{Modelling the Effects of Swirl}

The modelling described in 2.2 was expanded to include the ability to superimpose single vortex asymmetric swirl on top of the asymmetric and power law profile functions already implemented. The modified vortex equation from [4] was used to calculate a theoretical tangential velocity at a point in a vortex with a specified centre and radius. This is shown in Eq. (7).

$$
v_{\text {tangential }}=\frac{u_{0} r_{v} e^{-r_{v}^{2}}}{1-\frac{r}{R}+r_{c}}\left(1-\left[\frac{r}{R}\right]^{2}\right)^{\frac{1}{n}}
$$

To calculate the theoretical effect of swirl each path is divided into 10000 sub-lengths. To determine the swirl effect on each sub-length the x-component of swirl velocity from the above tangential swirl velocity was calculated. This is treated as a vector at 90 degrees to the axial velocity and vector addition is used to create a new combined velocity vector. Note that the sign of the $\mathrm{x}$ component is set according to whether the present point in the path is above or below the vortex centre. The remainder of the calculation proceeds as described in 3.2, the interception angle is calculated and used to calculate the component of velocity in the direction of the path. The sum of all such components is divided by the number of lengths to determine the average and converted to an axial velocity prediction by dividing by $\cos \sigma$. The results in this paper were calculated using this method.

The above method is fine for a chordal meter design in which there is no change in height across the path. However, if one wishes to model a different meter design, such as one incorporating bounce paths which requires 3 dimensions to represent, a new approach is needed. To do this the path is still divided into many sub-lengths but the swirl effect on each sub length is calculated by including the y-component of swirl velocity in addition to the $\mathrm{x}$ - 
Journal Pre-proof

component and by use of the dot product of the path vector $\mathbf{p}$ and the velocity vector $\mathbf{v}$. The result of the dot product is the velocity in the direction of the path weighted by the length of each path sub length. This is simply implemented because of the relation in Eq. (8):

$$
\boldsymbol{p} . \boldsymbol{v}=p_{x} v_{x}+p_{y} v_{y}+p_{z} v_{z}
$$

The $\mathrm{x}$ and $\mathrm{y}$ components of the velocity vector are found from the tangential swirl velocity and the $\mathrm{z}$ component of velocity is taken from the axial velocity profile function of your choice. The path vector components are constructed from a knowledge of the meter design, dimensions and number of sub-lengths. The result of $\boldsymbol{p} . \boldsymbol{v}$ for each sub length is summed which approximates the line integral across the velocity field and is then divided by the total path length to give a mean path velocity which forms the basis for the remainder of the calculation. This can be used for any meter design to test the appearance of swirl and asymmetry in varying proportions and to test for diagnostic correlations. For chordal designs, this is equivalent to the first approach described.

\subsection{Interference with Diagnostics}

Swirl changes the appearance of the profile and introduces additional errors, these could counter or add to the error already present from any axial asymmetry.

An illustration of the way swirl strength can change the appearance of the velocity profile is given below in Figs. 17A, 17B and 17C. These show single vortex swirl with varying vortex strength superimposed on top of profile A8. The swirl strength is varied from -0.2 to +0.2 in 0.1 increments simulating anti-clockwise and clockwise swirl for the parallel chord (Fig. 17A), the parallel crossed chord (Fig. 17B) and the in-plane crossed chord (Fig. 17C) arrangements. Note that as previously reported in [11] there is a significant difference in the appearance of swirl between the parallel chord and parallel crossed chord designs. A summary of the velocity profile diagnostics are given below each graph.

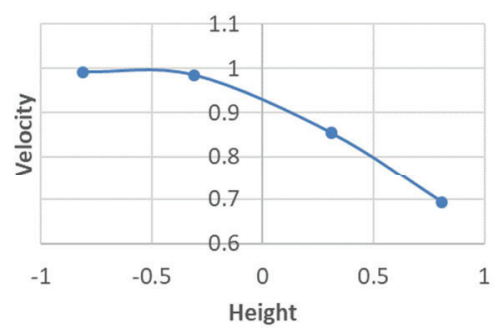

Profile factor 1.09, Symmetry 0.78 Possible swirl

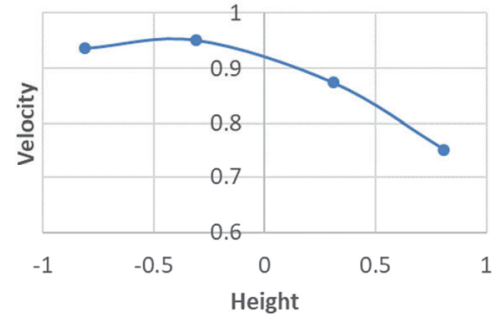

Profile factor 1.08, Symmetry 0.86 Possible swirl

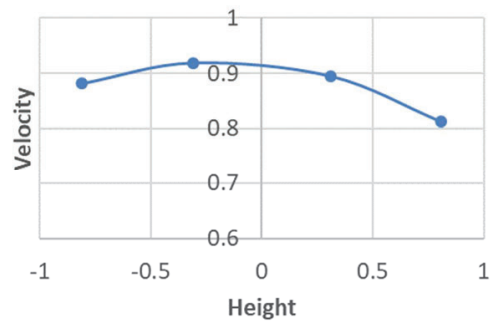

Profile factor 1.07, Symmetry 0.95 Possible swirl

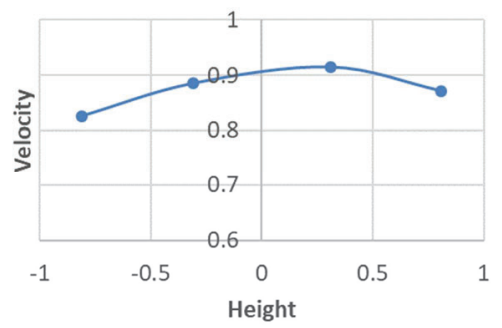

Profile factor 1.06, Symmetry 1.04 Possible swirl

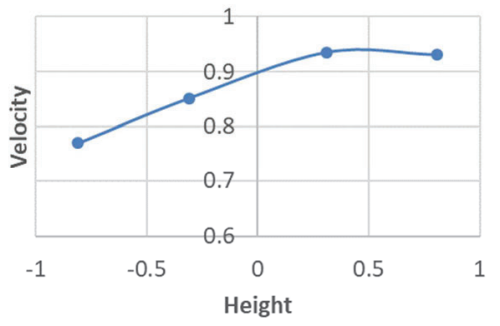

Profile factor 1.05, Symmetry 1.15 Possible swirl

Fig. 17A-Appearance of single vortex swirl with varying swirl strength and direction for parallel chord

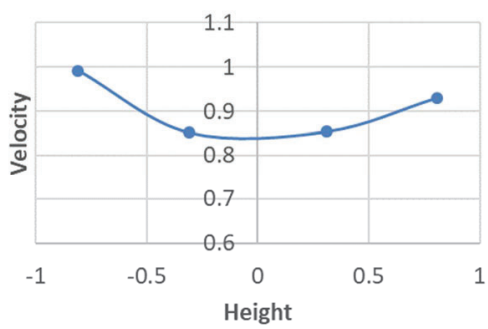

Profile factor 0.89, Symmetry 0.97 Possible swirl

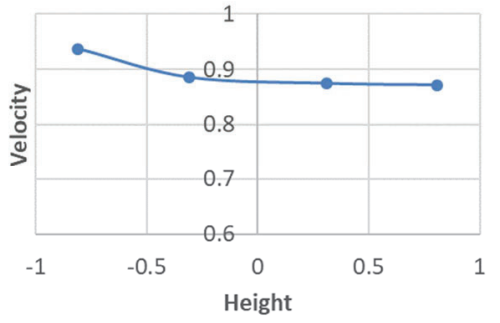

Profile factor 0.97, Symmetry 0.96 Possible swirl 


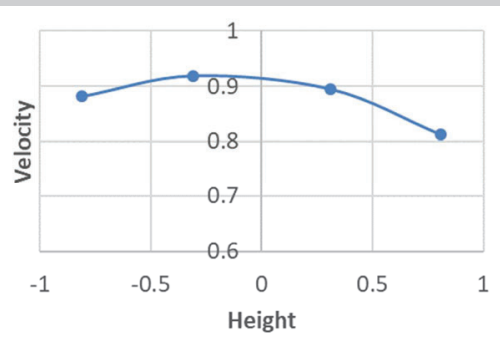

Profile factor 1.07, Symmetry 0.95 Possible swirl

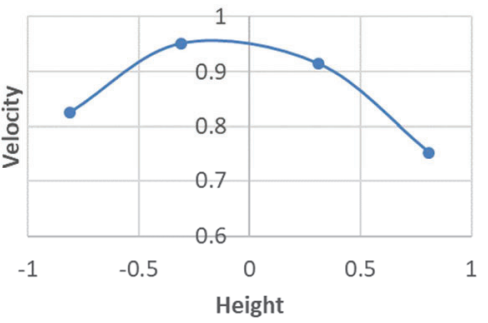

Profile factor 1.18, Symmetry 0.94 Possible swirl

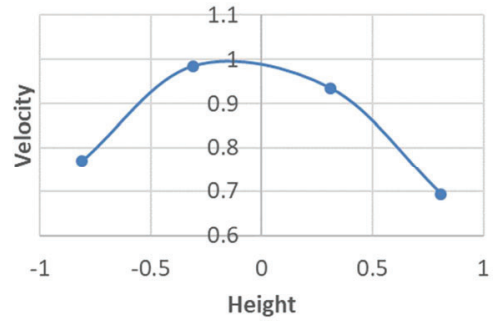

Profile factor 1.31. Symmetry 0.93 Possible swirl

Fig. 17B-Appearance of single vortex swirl with varying swirl strength and direction for parallel crossed chord

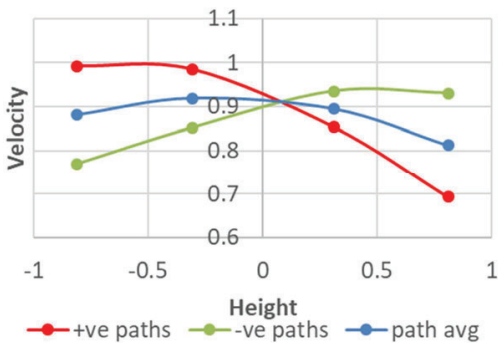

Profile factor 1.07, Symmetry 0.95 Swirl $-10 \%$

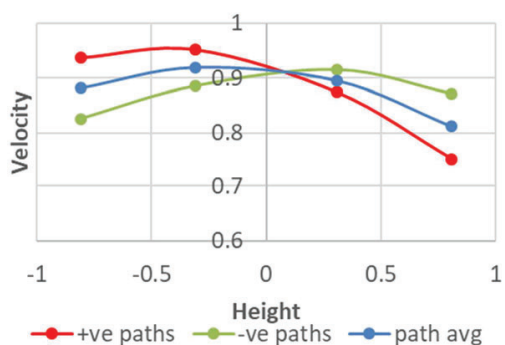

Profile factor 1.07, Symmetry 0.95 Swirl $-5 \%$

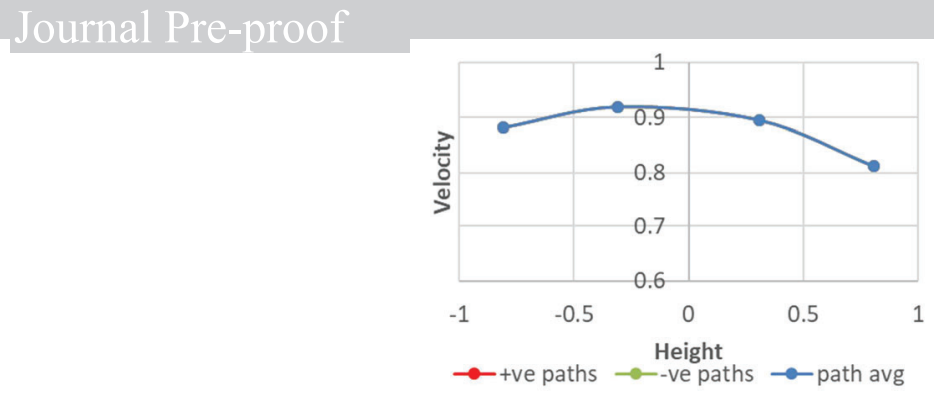

Profile factor 1.07, Symmetry 0.95

Swirl 0\% (note path avg is overlapping +ve and -ve paths)

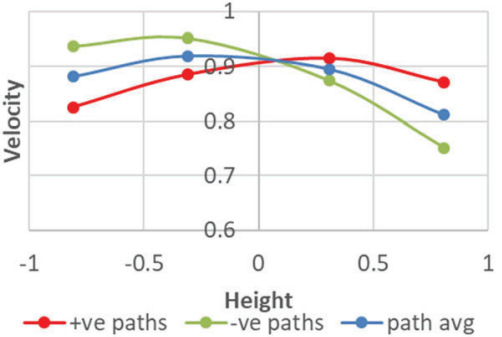

Profile factor 1.07, Symmetry 0.95 Swirl 5\%

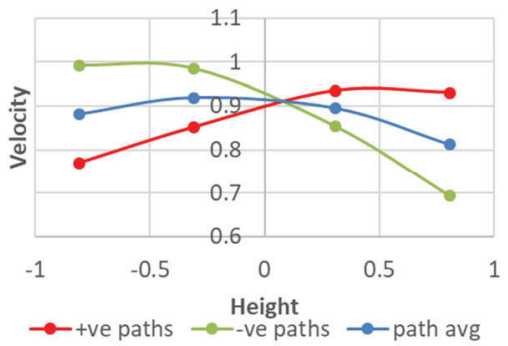

Profile factor 1.07, Symmetry 0.95 Swirl 10\%

Fig. 17C-Appearance of single vortex swirl with varying swirl strength and direction for in-plane crossed chord

When comparing the flow profile in service and at calibration we use established permissible changes in profile factor and symmetry ratio from the results of installation effect tests [2]. We can also use plots such as those in Figs. 17A-C to train ourselves to spot what swirl looks like for the meter configuration in question and therefore to help us identify the possible cause when a difference appears. However, if axial asymmetry is also in the profile this can easily cause us to misdiagnose the issue as will be shown shortly.

Relating a change in diagnostics to a change in error is extremely difficult at present and throughout the course of this work it became apparent that to begin to understand the magnitude of effect any difference is having on the measurement error two questions need to be asked: is swirl present and is axial asymmetry present. These are two separate effects with two separate changes in error for a given change in velocity profile diagnostics.

The charts in Fig. 18 were generated by running the model with asymmetric swirl superimposed on a power law profile with a varying vortex location across a square overlapping the centre at $-0.2 \leq x \leq 0.2$ and $-0.2 \leq$ $y \leq 0.2$. The square is divided into 10 columns and 10 rows. Each set of runs includes all vortex locations. For 
Journal Pre-proot

each set of runs one parameter was changed and the others held at the centre value in their range. There were 5 different values for swirl strength, swirl radius and swirl $\mathrm{n}$ (how the swirl diminishes at the pipe wall). These calculations were performed for both transducer configurations. The maximum swirl angle varies from approximately $2^{\circ}$ to $5^{\circ}$. The plots are based on profile factor and not symmetry ratio because no useful relationship between symmetry ratio and error was observed.
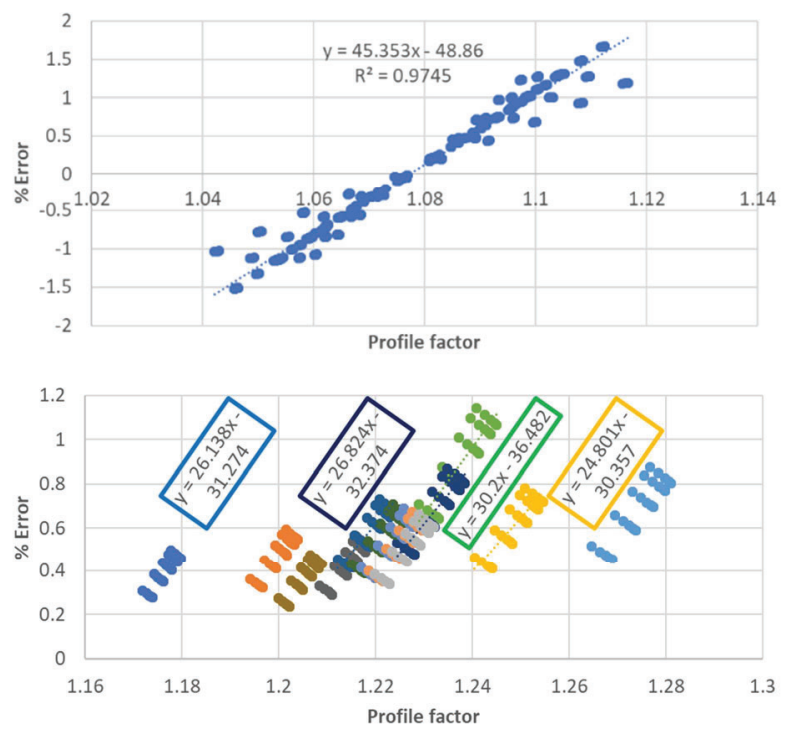

Fig. 18 - Error as a function of profile factor for parallel (top) and crossed 4 chord (bottom) arrangements

Fig. 18 shows that the parallel chord design does exhibit a strong correlation with profile factor $\left(r^{2} 0.97\right)$ in the presence of swirl alone and we can see that the sensitivity is around $0.45 \%$ change in error for a $1 \%$ change in profile factor caused by swirl.

It is more difficult to express the sensitivity to swirl for the parallel crossed chord design. From Fig. 18 the sensitivity appears less than the parallel chord due to the crossed chords partly cancelling the swirl but the swirl effect is still present because the chords are at different elevations. It is also evident there is not a single correlation. Each colour above represents a set of tests with varying vortex locations. The sensitivity due to vortex location is slightly different for each, but appears to be around $0.27 \%$ per $1 \%$ change in profile factor. The other swirl parameters also affected the error as expected, e.g. swirl strength and swirl radius but obvious correlations with profile factor or symmetry ratio were not evident.

In summary, it can be said that a small change in profile factor caused by swirl corresponds to a large change in error for both chord arrangements, that error being dependent on swirl position, strength and vortex size.

The purpose of showing this is to illustrate that a shift in profile factor caused by swirl is much more concerning than the same shift in profile factor caused by axial asymmetry. Axial asymmetry was shown earlier to have an effect of around $-0.018 \%$ per $1 \%$ change in profile factor (Gauss-Jacobi). Swirl appears to have an effect which can be far greater depending on the meter design and the swirl characteristics. It therefore follows that to better understand the potential effect on the measurement using present diagnostics we need to separate these two effects. The problem is that using the diagnostics with most current chordal designs it is difficult to separate what is swirl and what is axial asymmetry.

To illustrate this point further below are 3 meter designs measuring single vortex swirl superimposed on top of an asymmetric axial flow profile (A8). Figs. 19A, $19 \mathrm{C}$ and $19 \mathrm{E}$ represent a no swirl environment and are the base cases. Figs. 19B, 19D and 19F show the effect of swirl on these arrangements. The swirl strength in this example was 0.1 , swirl radius 1.6 , swirl $\mathrm{n} 15$, swirl vortex at $(0.1,0.1)$ and a meter radius of 1 . The resultant maximum swirl angle was $\sim 3.5^{\circ}$. The error due to axial asymmetry is specified as $\delta$.

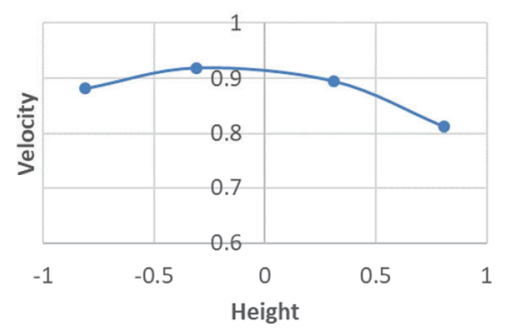

Fig. 19A - 4 parallel crossed chords no swirl ( $8 \%$ error, profile factor 1.07 , symmetry 0.95 )

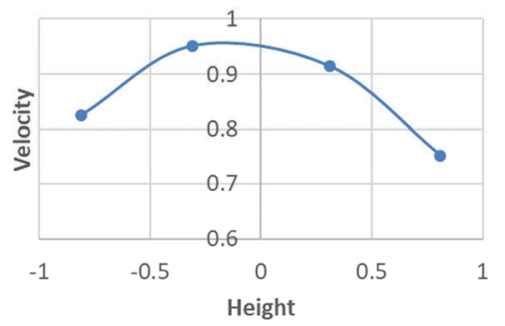

Fig. 19B - 4 parallel crossed chords with swirl $(\delta+0.4 \%$ error, profile factor 1.18, symmetry 0.94)

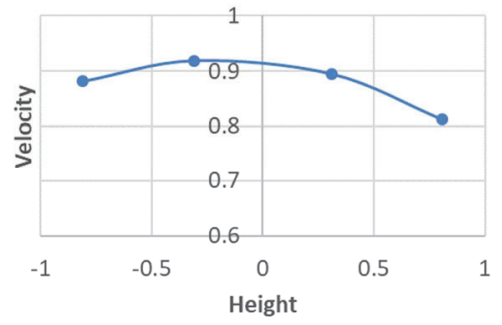

Fig. 19C-4 parallel chords no swirl ( $\delta \%$ error, profile factor 1.07 , symmetry 0.95 )

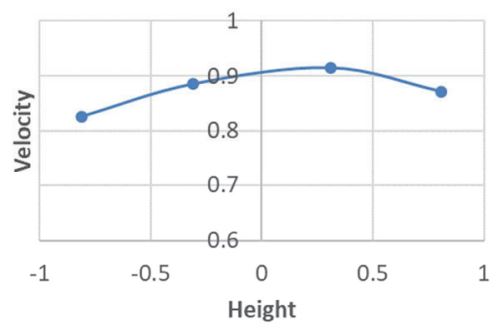

Fig. 19D - 4 parallel chords with swirl 


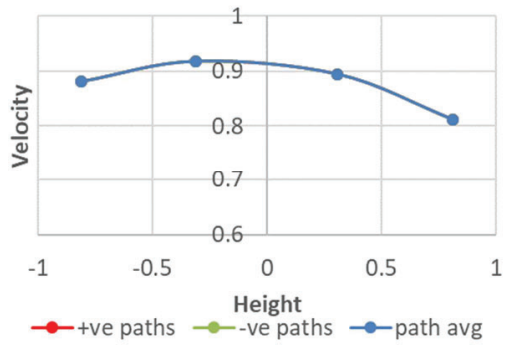

Fig. 19E - 4 in-plane crossed chords no swirl ( $\delta \%$ error, profile factor 1.07, symmetry 0.95. Note: path avg is overlapping +ve and-ve paths)

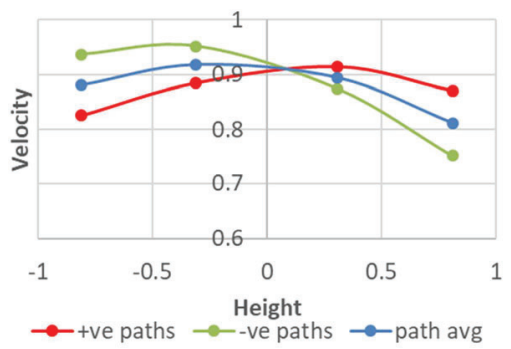

Fig. 19F - 4 in-plane crossed chords (8 paths) with swirl ( $\delta \%$ error, profile factor 1.07, symmetry 0.95 )

Consider Figs. 19B and 19D. How would the user know that swirl is in the profile and differentiate between axial asymmetry alone (which can influence the integration as shown above, the magnitude depending on the diagnostic corrections in place or number of chords) and a combination of axial asymmetry and swirl (which potentially can have a larger effect depending on the chord arrangement)?

Fig. 19F shows the in-plane crossed chord design in the same swirl scenario. However, we note that the average of the paths has recovered the underlying axial profile as per Figs. 19A, 19C and 19E. This allows for better understanding of the diagnostics. The user is no longer having to make a judgement call regarding what is swirl and what is axial asymmetry. This also presumably makes it easier to correct for the presence of axial asymmetry by diagnostic based corrections. A difference between the positive and negative paths at each chordal location is now apparent indicating the presence of swirl. The point at which the paths cross also indicates the vortex height.

It is theoretically possible for swirl to undo the appearance of axial asymmetry in the profile so all appears normal, but in fact potentially a significant measurement error can remain as illustrated in Figs. 20A and $20 \mathrm{~B}$ using profile $\mathrm{A} 1$.

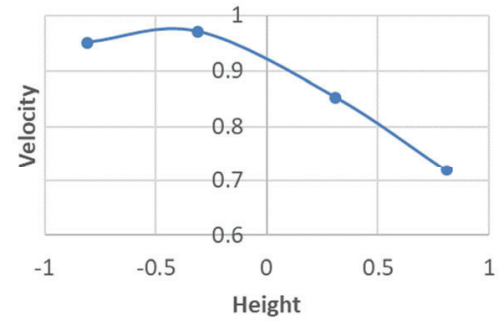

Fig. 20A - 4 parallel chords with axial asymmetry and no swirl ( $+0.39 \%$ error, profile factor 1.09, symmetry 0.82)

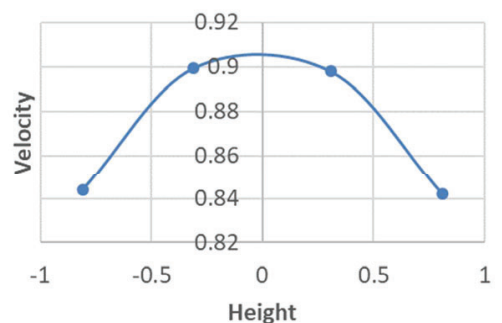

Fig. 20B - 4 parallel chords with swirl and axial asymmetry $(-0.47 \%$ error, profile factor 1.07 , symmetry 1.00 , swirl strength 0.19, swirl radius 1.29)

A similar case for the parallel crossed chord arrangement is one in which the true axial profile is symmetrical but excessively pointed with this being masked by swirl as shown using the power law in Figs. 21A and 21B.

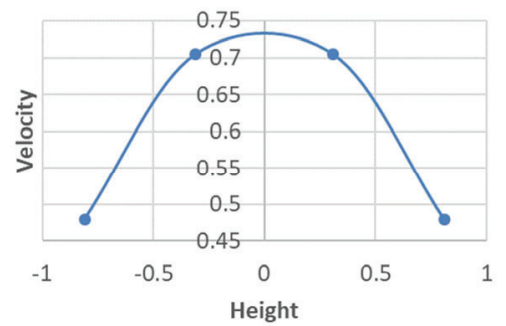

Fig. 21A - 4 parallel crossed chords with excessively pointed axial profile and no swirl $(+0.03 \%$ error, profile factor 1.47 , symmetry 1.0)

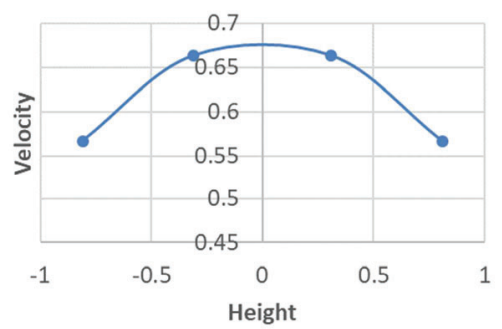

Fig. $21 B$ - 4 parallel crossed chords with excessively pointed axial profile hidden by swirl (-0.88\% error, profile factor

$$
\text { 1.17, symmetry 1.0) }
$$

Some research has looked to examine what correlations are evident from multiple calibrations and installation effect testing, for example [8]. In some cases, these tests will be on meters which have some automatic velocity profile diagnostic corrections included in the software. In those cases, the remaining error will depend on how well the diagnostic based correction has coped with the change in profile and interference from swirl. This is a reason the results may differ from those in this paper.

\subsection{Advantages of the In-Plane Crossed Chord Design}

The principal advantage of this design is swirl cancellation and consequent ability to perform to the required level in far from ideal situations. This has been well described in [2] in which it appears that the 8 path in-plane crossed chord design without a flow conditioner 
can outperform common 4 chord designs with a flow conditioner as shown in the results from the installation effect tests.

The addition of the flow conditioner obviously creates a pressure drop and a feature within the pipework to trap debris and cause problems with the measurement which has cost implications. The swirl cancellation and consequent lack of flow conditioner also suggests much smaller upstream straight length requirements. This was demonstrated in [2] which included the results of tests with 5D pipework upstream and is another cost saving feature. Some of the original promises of USMs were small footprint, large turndown and lack of pressure loss [9]. It appears this design does realise that goal given the minimal upstream straight lengths and lack of flow conditioner.

Swirl velocity measurement from first principles is a feature of the design and provides users with improved diagnostics. The measurement of swirl magnitude is also not affected by interference from axial asymmetry.

It appears that the permissible differences between the profile factor and symmetry ratio at calibration and those seen in the field are also larger with limits of $10 \%$ and $6 \%$ being used in [2]. This is because swirl and its associated interference with the diagnostics has been removed leaving only any possible integration error caused by axial asymmetry which is a lesser order effect.

A comparison of the profile factor and symmetry ratio at calibration against that seen in the field is often performed by the author on inspection and this is one of the great applications of velocity profile diagnostics. They allow us to confirm transferability of the calibration [9]. If the flow profile is excessively different in the field to that seen at calibration and the difference is stable this is concerning because this can lead to a persistent error in one direction. Such problems are often caused by a blocked flow conditioner, contamination of upstream pipework or they can be caused by installation effects. However as has been shown there is a degree of judgement involved as one must differentiate swirl from just axial asymmetry and to do that one must know what swirl looks like for the path configuration in question and base any judgement on the upstream pipework configuration as well. The situation would be far simpler for the in-plane crossed chord design. The swirl is removed from the measurement and we have the true underlying axial velocity profile, simplifying interpretation of the diagnostics. It then becomes a question of identifying what potential error there could be from the axial asymmetry which is possibly going to be one of the factors which will form the basis for competition between manufacturers.

We are fortunate at OGA to be exposed to a variety of measurement issues, including some with ultrasonic meter stations. These are usually identified using USM diagnostics and are often (but not always) velocity profile related. It seems prudent to ask of the errors seen, how many of these would have happened had this new design of meter been in use?

Of the 6 USM velocity profile issues we have been involved with in recent times 4 were caused by blocked flow conditioners. Assuming it would be used without a flow conditioner these are unlikely to have happened or had an effect. The contamination so far has been of the sort that would likely just pass through the meter without an issue (e.g. thin metallic strips, rust, fibrous compound used during welding) but it is feasible, however unlikely, that some contamination that might have been caught in the conditioner may go on to be caught on the USM body or damage it in some way and still cause an error.

Another was an installation effect caused by multiple out of planes bends and blank tees. In that case, the operator chose to stop using the 2 streams more affected by swirl and use the stream in which swirl appeared to not be present. There remains a noisy profile factor which is being monitored. Swirl cancellation would have removed the swirl issue so they would still have 3 streams, but it is difficult to say for definite whether the noisy profile factor is being caused by swirl and therefore whether this would be eliminated also.

Another was contamination of the upstream spool with glycol. Although luckily the USM escaped contamination (hence gains and VOS were unaffected) the presence of this substance and subsequent additional friction significantly changed the shape of the velocity profile to be much more pointed prompting an investigation. Upon inspection, it appears that if there was a measurement error (it may have coped with the change in profile given it was very symmetrical) it was likely an error in the integration due to the excessively different profile which would affect the in-plane crossed chord design in a similar fashion. Due to the contamination, the operator was unable to test the USM with the contaminated upstream spool so the actual error is unknown. It is also worth highlighting that initially there was concern that swirl could be present because an increase in profile factor along with a symmetrical profile could be caused by swirl for the path arrangement that was in use. This possible cause would have been immediately ruled out had the in-plane crossed chord design been in use because swirl measurement is a feature of the design.

In summary, it seems fair to say that of the 6 issues described 5 would have been significantly reduced or prevented using this design of meter.

\section{DISCUSSION AND CONCLUSIONS}

In short using more than 4 paths with chordal USMs can yield a very significant improvement in the integration if the paths are used in a meaningful way. The assertion in [1] that using more than 4 paths has little effect on the integration has been explored considering two possible meanings.

If we assume 'path' means 'chord' whilst it is noted that the results reported were repeated for one type of Gaussian quadrature and a symmetrical power law profile it has been shown that this is not the case when asymmetry in the axial profile is considered with reductions in error span of $0.2 \%-0.3 \%$ and reductions in average absolute error of $\sim 0.15 \%$ being typical. This is also not true for a symmetrical power law profile for all types of Gaussian quadrature, and it was shown in the above that there was a larger increase in performance for Gauss-Legendre with more chords on the power law profile. 
Toumal Pre-proot

If we assume the literal meaning of 'path' it has been shown this is not the case. The theory behind extra paths for the purposes of swirl cancellation has been explained and results from installation effect tests show the principle works not just in theory but in practice and offers significant performance benefits and cost savings for operators.

Combinations of swirl and axial asymmetry interfere with our interpretation of the diagnostics and make it harder to identify whether swirl is or isn't present and to quantify the error being caused by a shift in the diagnostics. The in-plane crossed chord design simplifies this problem by it being a question of determining the error due to axial asymmetry only. The magnitude of residual errors will depend on whether diagnostic corrections are in use, how strong the relationship is and the number of chords used in the design.

Just like conventional 4 chord designs, the in-plane crossed chord design still requires correction for the presence of axial asymmetry to achieve optimal performance. Because of this fact operators using this meter still need to monitor for differences between the flow profile at calibration and that seen in service. It does appear at present that the permissible differences in profile factor and symmetry ratio are potentially wider, and the potential errors once outside these limits are potentially smaller. For users (OGA are included as a user in this regard) to make a judgement on what is an acceptable difference and what is not the data from installation effect tests (e.g. those specified in ISO-17089 [13] or OIML [14]) needs to be released by the manufacturer. Without this information users will be forced to adopt more cautious permissible differences and the true value of these meters may not be realised.

The profile factor correlation for the purposes of axial asymmetry correction has been reviewed. By use of the interpolation polynomial we have seen why this correlation appears to work. It was found that in general more chordal locations does not improve the correlation.

The modelling demonstrates that a given change in profile factor caused by swirl has a larger impact on the measurement than the same change in profile factor caused by axial asymmetry. This suggests that in the case where both axial asymmetry and swirl are present concurrently in unknown proportions in the meter bore and swirl cancellation is not in use, a change in velocity profile diagnostics cannot be used to estimate the magnitude of the error.

Diagnostic corrections for axial asymmetry and including more chords in the design are two different ways of achieving the same thing, a reduction in sensitivity to axial asymmetry. Meters with more chords or with diagnostic based corrections are likely to be more resilient to changes in the axial profile (however they can be fooled in the presence of swirl without swirl cancellation). Such changes could occur when moving from a calibration facility to the field, where multiple bends or tees could be distorting the profile. Using diagnostic based corrections or more chords is therefore a way to reduce the installation effect bias. It is often referred to as an uncertainty, however OGA would only consider it to be true uncertainty if the difference between the calibration and service profiles is constantly changing with the errors being evenly distributed around 0 . In the authors experience these differences are often stable which would yield a persistent integration error in one direction, and therefore a bias.

However, one could make the argument that if a diagnostic based correction can yield the same performance as more chords, why bother producing a more expensive meter with more chords? The answer to this is as follows.

At present the detail behind these corrections, how they were derived and how they are applied, is not usually divulged. In some cases, users do not know what errors remain after application of these corrections, the only insight is available from the installation effect testing if this data is released. The corrections are slightly different between each of the profiles and (assuming this is also the case in real profiles) this presumably creates differences in the way they are applied between manufacturer. We have also seen the potential for swirl to interfere with the diagnostics, making it harder still to apply an appropriate correction (where the in-plane crossed chord design principle is not used). This gives a little insight into the complexity involved in applying a diagnostic based correction and what potential there is for them to differ between manufacturer.

The use of confidential diagnostic based corrections also presents a problem for anyone trying to model the meter performance. Users sometimes use modelling when trying to justify the expense of changing an installation. In the present day, it is rarely enough to say expense must be incurred to correct some deficiency without an approximate quantification of the error being caused. We can use results from installation effect tests and these are very useful but unless the installation and meter type matches up to the test perfectly it may not be relevant. Without knowledge of the detail of the corrections the user can't predict the meter response from modelling, even with a perfect CFD model. There is concern that not including these effects in models could lead to a false positive, for example recommend installation of a flow conditioner when in fact it is not required (the author has seen one example where this may have happened) or a false negative, where the model predicts the meter should cope when in fact, due to the corrections being used in situations for which they were not developed, performance is being adversely affected.

Using more chordal locations may change the above problem. If the integration scheme in use is known (i.e. weights and chordal locations which some manufacturers do publish) the user has a chance to model the meter if the CFD simulation is accurate. The operation of the meter is also more transparent.

\section{KEY POINTS}

\section{Operators}

4 chords are not optimal. As meters develop and more chords are introduced these will make the meter more resilient to asymmetry in the axial velocity profile but this does not help them combat swirl. This will also mean any confidential diagnostic corrections will have less of an effect because the effects of axial asymmetry have been addressed using a first principles approach. 
Journal Pre-proot

4 paths are not optimal. Extra paths crossed on the same chord for swirl cancellation (the in-plane crossed chord design) is a major performance enhancement and results in various cost saving features such as reduced footprint due to the reduced upstream straight length requirements and no requirement for flow conditioning which reduces the likelihood of mismeasurement due to blockages and simplifies the calibration process. The design also simplifies the interpretation of velocity profile diagnostics due to the absence of any interference from swirl.

Whilst the in-plane crossed chord design is not affected by swirl it is still affected by axial asymmetry so monitoring for differences between the velocity profile seen at the calibration facility and that seen in service is still required. Be aware that some manufacturers may not include automatic diagnostic based corrections and ask to see the results from the installation effect tests. The results from these tests should illustrate permissible profile factor and symmetry ratio changes that should result in minimal error in the measurement.

Meters that use automatic velocity profile diagnostic corrections for axial asymmetry are likely to be more resilient to changes in the axial velocity profile than the same path configuration and quadrature type which doesn't include a correction unless they use more than 4 chords.

When modelling the meter response using CFD any velocity profile diagnostic corrections should be included in the model otherwise there is a real risk the predicted measurement error will be inaccurate.

\section{Manufacturers}

There are a few manufacturers now producing the inplane crossed chord design. The design is a worthy addition to a product portfolio.

Consider whether more chords could be added because this will improve the meters ability to handle changes in the axial velocity profile. If diagnostic based velocity profile corrections are in use this will also reduce reliance on these and allow users to better model the meter response.

The data from installation effect tests (such as those performed for ISO 17089 [13] and OIML R137 [14]) should be released for different meter types to help users decide what is and what isn't an acceptable change in velocity profile diagnostics.

\section{$\underline{\text { Regulators \& Auditors }}$}

The theory behind the in-plane crossed chord design and test data released so far suggests that these meters can perform to the required level without a flow conditioner and with minimal upstream straight lengths and this represents a significant opportunity for cost savings.

Monitoring for differences between the flow profile at calibration and that seen in service is still required for the in-plane crossed chord design because it will be affected by axial asymmetry to a lesser or greater degree, the magnitude of the effect depending on whether velocity profile diagnostic corrections are in use and the number of chords.

When reviewing results from CFD simulations query whether the presence of any diagnostic based velocity profile correction has been accounted for because it can affect the predicted measurement error.

Manufacturers should be encouraged to release data from installation effect testing to ensure maximum value from their meters is realised and to help establish permissible differences in velocity profile diagnostics for each meter model.

Modelling the effects of axial asymmetry and swirl on USMs helps improve understanding and provides a platform to test designs and diagnostic correlations. 


\section{NOTATION}

$A_{k} \quad$ weight for chordal location $k$

$c \quad$ velocity of sound in fluid

$f(x)$ chordal line integral function

$L \quad$ path length

$L(x) \quad$ chord length at $x$

$l_{k}(x) \quad$ Lagrange polynomial

$n$ parameter controlling swirl behaviour at pipe wall

$P(x) \quad$ polynomial approximating $f(x)$

$R \quad$ meter radius

$r \quad$ current radial position

$r_{c} \quad$ distance from vortex center

$r_{0} \quad$ vortex radius

$r_{v} \quad r_{c} / r_{0}$

$\bar{V} \quad$ mean fluid velocity

$v \quad$ axial velocity

$\bar{v}(x) \quad$ mean chordal velocity at $x$

$w(x) \quad$ weight function

$x_{k} \quad$ height at chordal location $k$

$\sigma \quad$ path angle

\section{ACKNOWLEDGEMENTS}

I would like to express my gratitude to Dr. Gregor Brown for his expertise, support, guidance and valued feedback during development of the model and throughout the course of this work.

I would like to thank Douglas Griffin for his support during this work, for his review and valued feedback.

I would also like to thank Steve Peterson, Phil Stockton and Dr. Ghazi Droubi for taking the time to review parts of this work and for providing valuable feedback.

I am also very grateful to the reviewers from the Journal of Flow Measurement and Instrumentation and thank them for their feedback and suggestions.

\section{REFERENCES}

[1] K. J. Zanker, T. Mooney. Limits on achieving improved performance from gas ultrasonic meters and possible solutions, NSFMW 2012.

[2] G. J. Brown, W. R. Freund. Analysis of diagnostic data from an 8-path ultrasonic meter, AGA Operations Conference May 2014.

[3] P. I. Moore, G. J. Brown, B. P. Stimpson. Ultrasonic transit-time flowmeters modelled with theoretical velocity profiles: methodology, Measurement Science \& Technology. Volume 11 (2000).

[4] G. J. Brown, H. Estrada, D. Augenstein, T. Cousins. Ultrasonic metering of liquefied natural gas for allocation and custody transfer, SE Asia FMW March 2007.
[5] K. J. Zanker. The effects of reynolds number, wall roughness and profile asymmetry on single and multipath ultrasonic meters, NSFMW 1999.

[6] G. J. Brown. Factors affecting the performance of ultrasonic flow meters, NSFMW 1997.

[7] Keisan online calculator, https://keisan.casio.com/menu/system/000000001110

[8] R. Miller, E. Hanks. Gas ultrasonic meter installation effects and diagnostic indicators, NAFFMC.

[9] K. J. Zanker, T. Mooney. The transit time difference ultrasonic gas meter - a reassessment, NSFMW 2003.

[10] J. Yoder. The Path to Chordal Harmony, www.flowresearch.com

[11] G. J. Brown, W. R. Freund, A. McLachlan. Testing of an 8-path ultrasonic meter to international standards with and without flow conditioning, AGA Operations Conference May 2013

[12] BS 7965:2013. Guide to the selection, installation, operation and calibration of diagonal path transit time ultrasonic flowmeters for industrial gas applications.

[13] ISO 17089-1:2010. Measurement of fluid flow in closed conduits - ultrasonic meters for gas - Part 1: Meters for custody transfer and allocation measurement.

[14] OIML R 137-1\&2:2012. Part 1: Metrological and technical requirements. Part 2: Metrological controls and performance tests. 
Highlights from "An Investigation into the Performance and Diagnostics from Different Chordal Integration Schemes in Asymmetric Flow"

- 4 chordal locations do not appear the optimal configuration for chordal ultrasonic flow meters as has been previously asserted. More chords allow the integration scheme to perform better in the presence of axial asymmetry.

- The interpolation polynomial upon which the gaussian quadrature in use is based can be used to visualise why integration errors occur in axial asymmetry.

- The correlation between profile factor and error in the presence of axial asymmetry and no swirl does vary between different axial velocity profiles.

- Combinations of axial asymmetry and asymmetric swirl can in theory cancel each other out and appear as normal velocity profiles in the diagnostics of some meter designs but a significant error remains.

- It is shown that a change in profile factor caused by swirl has a much larger effect on the measurement than the same change caused by axial asymmetry and that in some designs where non-axial velocity components are present this means traditional velocity profile diagnostics cannot be used to infer the measurement error from a change in diagnostics. 


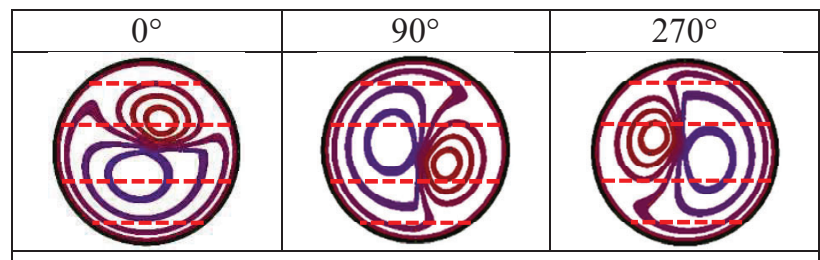

Table 1 - Rotation of the velocity profile (dashed lines indicate the chordal paths) 